\title{
In search of lost time - a case of myocardial perforation in a patient with arrhythmogenic right ventricular cardiomyopathy
}

\author{
Carina Ureche*, Radu A. Sascău, Cristian Stătescu \\ Institute of Cardiovascular Disease "Prof. Dr. George I.M. Georgescu", lasi, "Grigore T. Popa" \\ University of Medicine and Pharmacy, lasi, Romania
}

\begin{abstract}
Arrhythmogenic right ventricular cardiomyopathy (ARVC) is particularly hard to diagnose and manage. We present the case of a 49-year-old, a former professional football player, with a history of cardiac arrest in 2011 by ventricular tachycardia (VT) (normal coronary arteries). Between 2011 and 2019 the patient didn't present for reevaluation and resumed endurance sports activity. In 2019 he was directed to our clinic for a syncope followed by constrictive anterior chest pain and palpitations, the clinical expression of a VT for which cardioversion was required. Upon admission, the patient was at sinus rhythm with negative T waves in V1-V2. Echocardiography showed significant dilatation and dysfunction of the RV (TAPSE $16 \mathrm{~mm}$, FAC $20 \%$, S' 8.6 $\mathrm{cm} / \mathrm{s}$ ). To confirm the diagnosis of ARVC, cardiac MRI was performed, confirming fat infiltration in the RV free wall with biventricular involvement. Given the high arrhythmic risk, a two-chamber ICD was implanted. In the second postprocedural day, the patient presented important epigastric pain, with ECG signs of sensing and pacing malfunction and ventricular probe displacement on the radioscopy. Emergency surgery was performed, with successful extraction of the electrode. Postoperative progression was favorable under treatment with betablocker and amiodarone. In conclusion, this case is a particular one since we've documented two distinct phases in the evolution of the ARVC (electrical phase - 2011 and structural phase - 2019). Moreover, by resuming endurance sports activity and in concordance with the literature data, we can only assume that the progression of the disease was accelerated, with a greater arrhythmic risk.
\end{abstract}

Keywords: arrhythmogenic right ventricular cardiomyopathy; ventricular tachycardia; echocardiography; implantable cardiac defibrillator; lead perforation

\section{Introduction}

Arrhythmogenic right ventricular cardiomyopathy (ARVC) is particularly hard to diagnose and manage. Its main characteristic is the progressive replacement of viable myocardium with adipose tissue, predominantly at the level of the right ventricle.

Received: October 2019; Accepted after review: December 2019; Published: December 2019.

${ }^{*}$ Corresponding author: Carina Ureche, Institute of Cardiovascular Disease "Prof. Dr. George I.M. Georgescu", lasi, "Grigore T. Popa" University of Medicine and Pharmacy, Iasi, 16 Universitatii Street, Romania.

Email: carina.ureche@gmail.com
Biventricular involvement is also possible and encompasses a worse prognosis. ARVC is a genetic disease, but physical exercise can be a contributing factor and may accelerate the progression of the disease in patients carrying the desmosome mutations. Since it carries a high arrhythmic risk, most often patients are diagnosed when they express the electrical phase - most common by ventricular arrhythmias. An implantable cardioverter defibrillator is recommended in this setting, but given the important changes in the ventricular walls (intense trabeculation, aneurysms), the procedure can be challenging. 


\section{Case presentation}

We present the interesting case of a 49 years old male, an ex-professional football player, referred to our clinic with the suspicion of acute coronary syndrome, after a syncope followed by constrictive thoracic pain and palpitations.

He was known to our clinic since 2011 (8 years ago), when he suffered a cardiac arrest after being hit in the chest by a football during a football match. He was resuscitated on the field, requiring 10 external electrical shocks for an incessant ventricular tachycardia (VT). After reestablishment of the sinus rhythm, he was addressed to our clinic for further investigation. Coronary angiography showed normal coronary arteries. Taking into consideration the young age, the morphology of the VT (left bundle branch block morphology - right ventricle (RV) origin and superior axis) and the mild dilation of the RV, a suspicion of ARVC was raised. Electrophysiological study was performed, but no ventricular arrhythmias were further induced. The patient was started on amiodarone and reevaluation with cardiac
MRI was recommended for the confirmation of ARVC. Furthermore, complete cessation of professional football was advised. Despite this, between 2011 and 2019 the patient did not return for reevaluation, stopped any medical treatment and resumed the endurance physical activity.

In February 2019 he presents a syncopal episode while undergoing regular house activities, followed by constrictive thoracic pain and palpitations. A suspicion of acute coronary syndrome was raised, so the patient was referred to our clinic. During transportation, two episodes of sustained VT were recorded, requiring two electrical shocks and intravenous amiodarone, with reestablishment of sinus rhythm.

On admission in our department, he was hemodynamically stable, with normal blood pressure and heart rate, without angina. No cardiac murmurs or pulmonary rales were found on auscultation.

The electrocardiogram showed sinus rhythm $60 \mathrm{bpm}$, sequelae of anterior myocardial infarction, negative $\mathrm{T}$ waves in $\mathrm{V} 1$ V2 (Figure 1.)

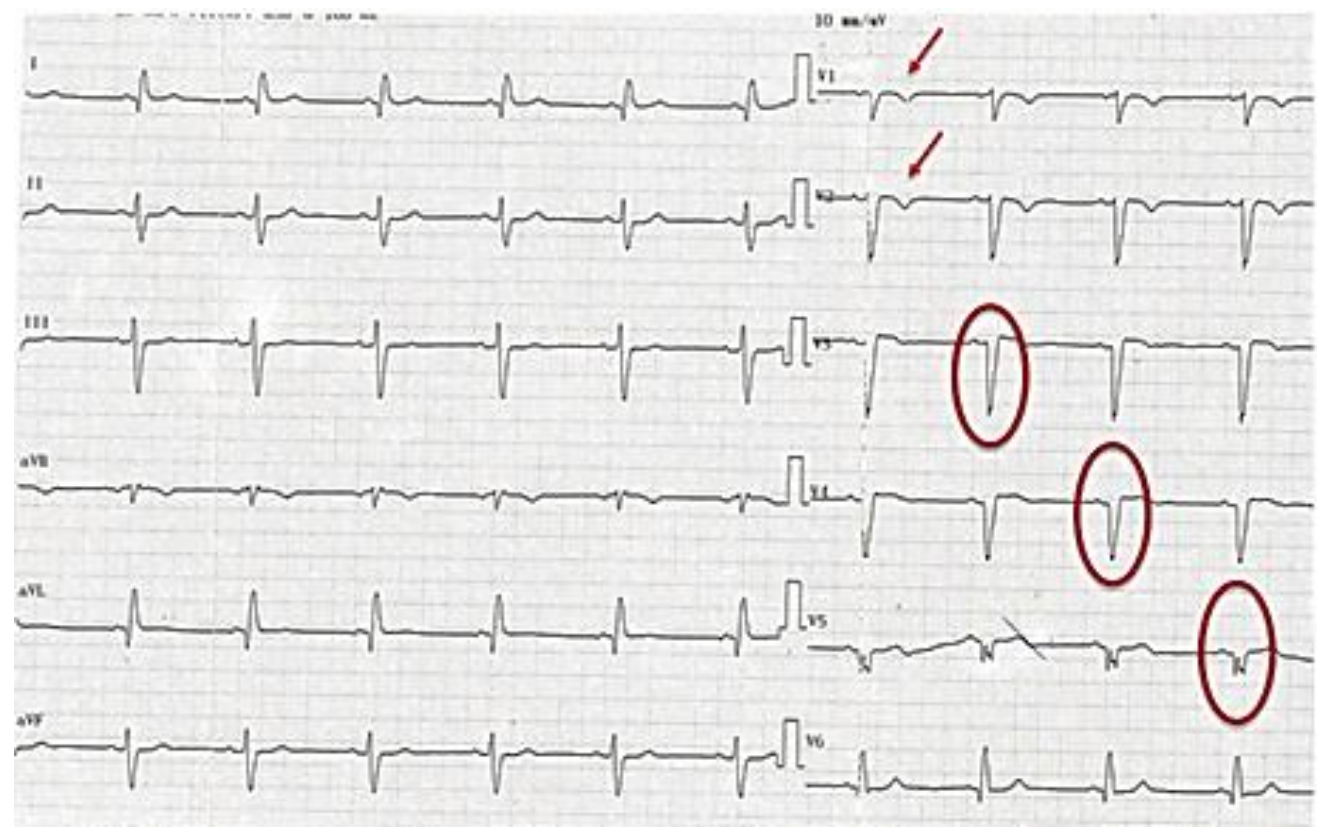

Fig. 1. ECG: SR $60 \mathrm{bpm}$, negative T waves in V1-V2, QS in V3-V5

By analyzing the ECG trace from the ambulance, a wide complex tachycardia with a left bundle branch block morphology and inferior axis was observed, suggesting a VT originating in the RVOT.
The blood tests revealed hypopotassemia (3.4 $\mathrm{mmol} / \mathrm{l})$, mild dyslipidemia, normal myocardial and hepatic enzymes, normal TSH.

At echocardiography we found an extremely dilated RV, with dyskinesia of the 
apex and apical half of the RV free wall, with aneurisms at this level. A focused examination of the RV showed a dilated RVOT measured both in PLAX (32 mm) and PSAX (41 mm), with a severely reduced systolic function fractional area change $20 \%$, TAPSE $16 \mathrm{~mm}$,
$S^{\prime}$ velocity $8,6 \mathrm{~cm} / \mathrm{s}$. The $\mathrm{LV}$ was non dilated, with a normal ejection fraction and without kinetic abnormalities but with a small degree of trabeculation at the apical level. No signs of aortic dissection or significant valvopathies were found (Figure 2).
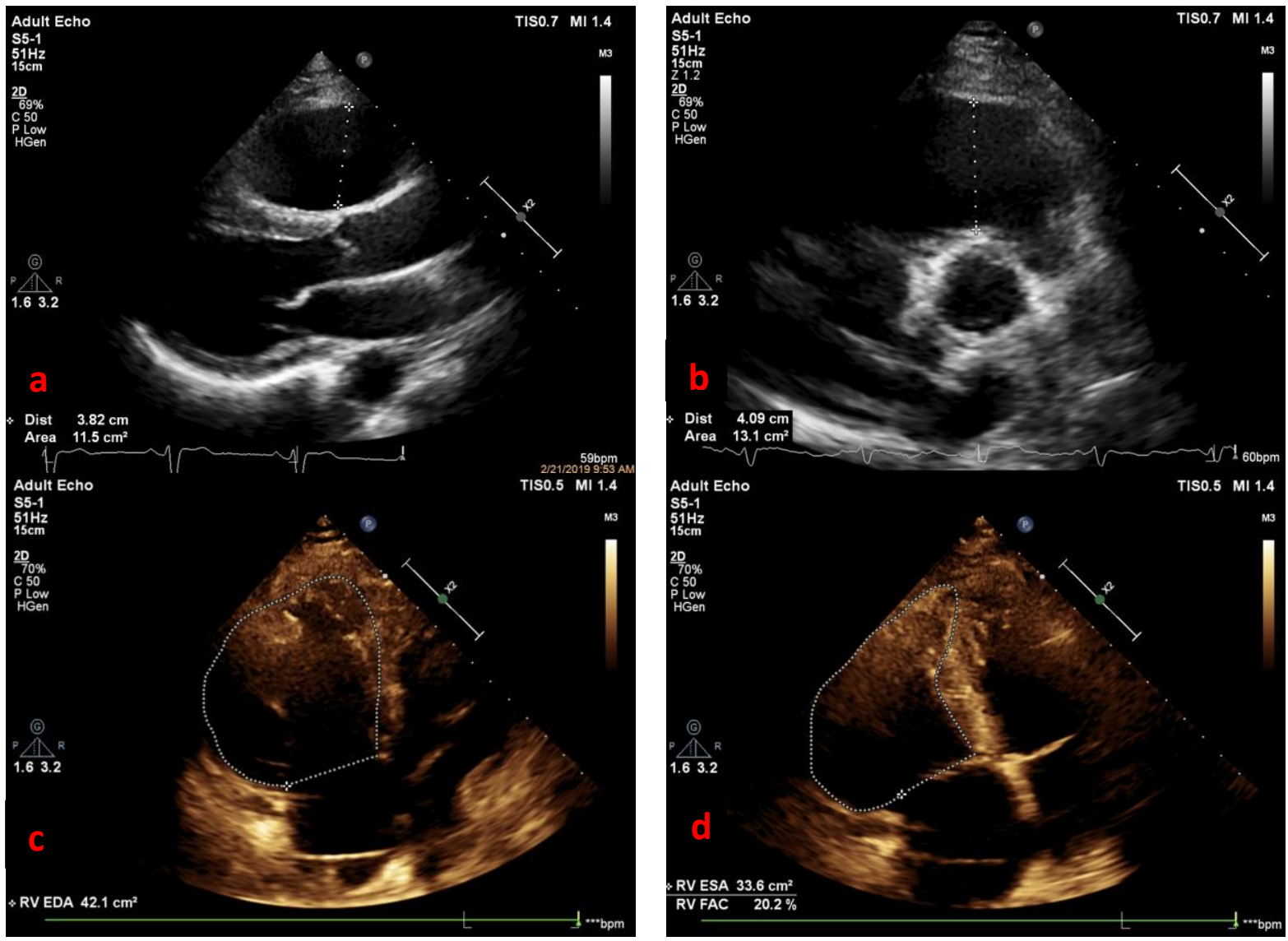

Fig. 2. Echocardiography: Dilated RVOT (PLAX - a, PSAX - b) and decreased FAC (c, d).

Chest $X$ ray was normal. In order to exclude an ischemic etiology of the VT, we decided to perform a coronary angiography, which was normal.

At this moment, the differential diagnosis of ARVC included myocarditis, sarcoidosis, idiopathic RV VT, athlete's heart, excluded by the normal LV function and walls without hypertrophy, normal myocardial enzymes and chest $X$ ray.

In order to confirm our suspicion and further risk stratification, we decided to perform a cardiac MRI, which showed an extremely dilated RVOT (43 mm) and RV (EDV/BSA $223 \mathrm{ml} / \mathrm{m} 2$ ), with aneurisms and hypokinesia, dyskinesia and zones of akinesia located at the level of the RV free wall (Figure $3)$. The systolic function of the RV was found to be severely depressed - RV ejection fraction $26 \%$, global longitudinal strain $-7 \%$, global circumferential strain $-4 \%$. Furthermore, inhomogeneous adipose infiltrations of the RV free wall and at the apical segment of the lateral wall of the LV, with extensive fibrosis at this level were observed (Figure 4).According to the 2010 Task Force Criteria for diagnosis of ARVD, the patient had 3 major criteria and 2 minor criteria, establishing a certain diagnosis [1]. 

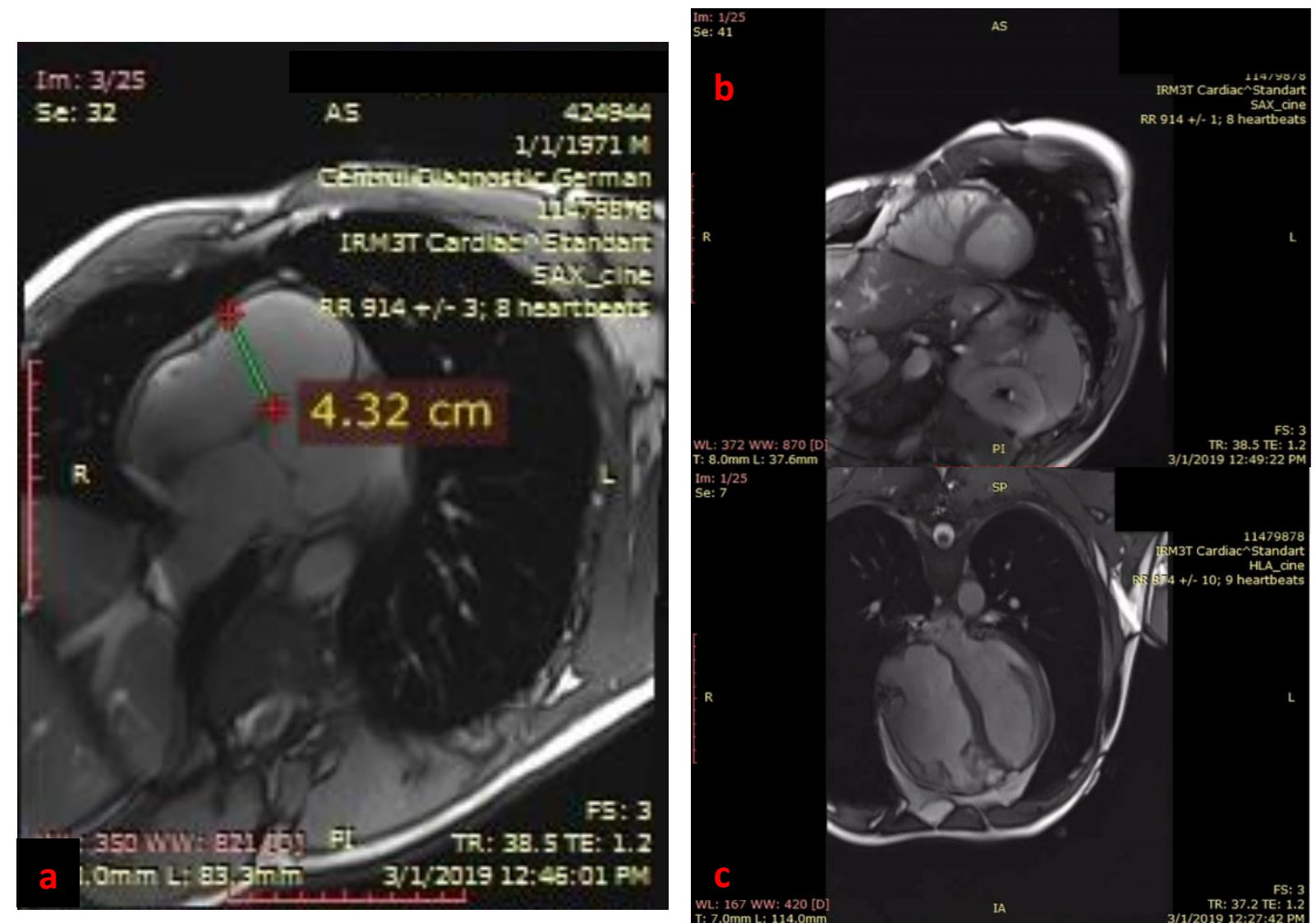

Fig. 3. Magnetic resonance aspect: dilated RVOT (a) with aneurisms and hypokinesia, dyskinesia and zones of akinesia located at the level of the RV free wall (b). Inhomogeneous adipose infiltration of the RV free wall, with extensive fibrosis that also involves the left ventricle (c).

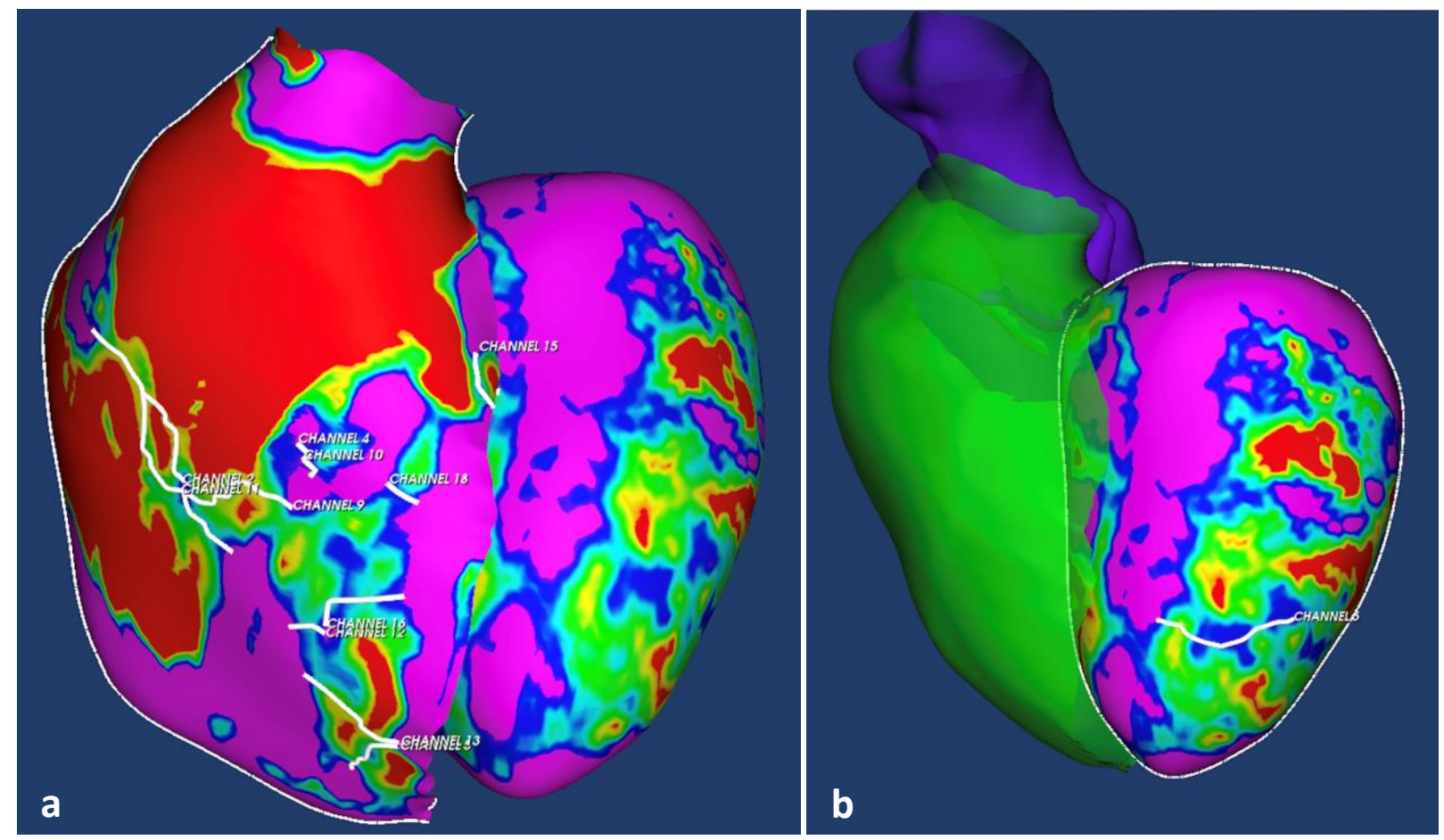

Fig. 4. Extensive fibrosis at biventricular level $(a, b)$. 
Repeating the electrophysiological study and further ablation of the arrhythmogenic substrate was considered. Given the possible epicardial and endocardial substrate, the extensive, inhomogeneous fibrosis lesions at biventricular level, the intense trabeculation and parietal aneurisms, and the fact that in our center there is no epicardial approach available, we decided that the risks outweigh the possible benefits of the procedure.

Taking all of the above into consideration and according to the risk stratification system recommended by the ESC/AHA [2], our patient was found to be at the highest arrhythmic risk (8-10\%/year), with a class I indication for implantable cardioverter defibrillator. The implantation was performed without immediate periprocedural complications.

At 48 hours after the implantation, the patient developed atrocious epigastric pain, with no response to routine pain killers. An emergency ECG was performed, showing pacing and sensing malfunction and the echocardiography showed no pericardial fluid. The abdominal ultrasound showed no free fluid in the abdominal cavity and no signs of acute abdominal emergency. The thoracic radioscopy showed displacement of the ventricular probe, suggesting a myocardial perforation (Figure 5).

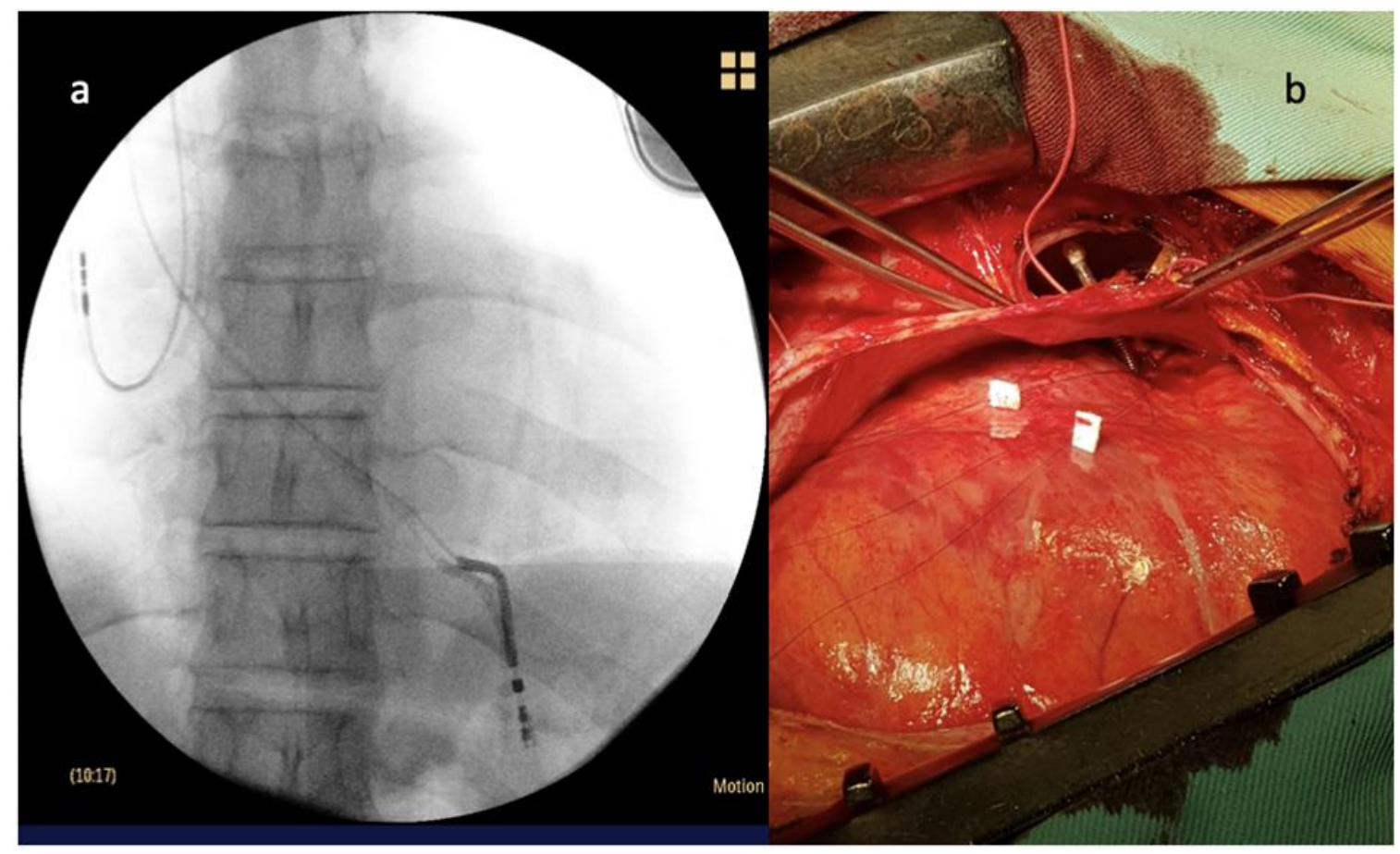

Fig. 5. Displaced ventricular probe - (a) radioscopy and (b) intraoperative aspect.

Given the severe alteration of the patients' status and high risk, emergency thoracotomy with successful withdrawal of the ventricular probe was performed. The myocardial perforation was then sutured.

The postoperative evolution of the patients was slowly favorable, without repeating the ventricular arrhythmias. He was discharged after 14 days and treatment with a beta blocker, amiodarone, spironolactone and atorvastatin was recommended. As future perspectives, the patient will be referred for evaluation in a center where epicardial approach for ablation is feasible and implantation of a subcutaneous defibrillator will be performed as soon as the device will be available.

\section{Discussions}

ARVC is a disease first described in the 1970s in patients undergoing ablation for RVOT ventricular tachycardia. Since then, several cases and series of cases have been published leading to the inclusion of this entity 
in the spectrum of cardiomyopathies in 1994. Nowadays, clear diagnostic criteria are available in order to ease the early diagnosis of this disease [2]. The natural history of this disease includes four stages - the "hidden" phase (asymptomatic patient but at high risk for sudden cardiac death), the electrical phase (when ventricular arrhythmias occur), the structural phase (with modifications in the structure of the RV due to fat infiltration) and the advanced phase (evolution towards dilative cardiomyopathy and severe dysfunction) [2]. In our case, the suspicion of ARVC was first raised in 2011, when the patient presented with VT, but did not meet the criteria for ARVC diagnosis (the electrical phase) and was confirmed 8 years later in the structural phase. This underlines the importance of having a high level of suspicion regarding this condition in young patients with minimal to no RV enlargement and VT originating from the RV.

Moreover, in patients carrying a desmosome mutation (which are proved to be associated with the development of ARVC), exercise was related with a higher risk of developing malignant arrhythmias and progression to dilative heart failure [3]. This lead to the recommendation that patients with a definite diagnosis of ARVC should not participate in competitive and/or endurance sports (Class I) and should restrict athletic activities, with a possible exception: recreational low-intensity sports (Class Ila). In our case, by resuming endurance sports activity and in concordance with the literature data, we can only assume that the progression of the disease was accelerated and the arrhythmic risk was greater [2].

Ablation can be considered for this category of patients, but given its progressive pattern, it's often necessary to repeat the procedure. Also, there is a high risk of complications related to the endocardial approach, given the modifications of the walls, with intense trabeculation and aneurysms. In centers were epicardial approach is available, this could represent a good alternative [4].
ICD implantation has a class I indication for high risk ARVC patients (aborted sudden death, hemodynamically unstable sustained VT, severe RV/LV dysfunction), a class Ila indication for intermediate risk patients with $>1$ major risk factor: syncope, NSVT, moderate dysfunction of the RV/LV) and a class Ilb indication for those without [5]. After implantation, it was shown that approximately $80 \%$ of the patients develop at least one episode of VT/VF, and $20 \%$ die, in spite of the ICD [6]. The problem with ICD implantation in ARVC patients arises from the high rate of postprocedural complications (reported around $60 \%$ ), with almost half of them being leadrelated [6]. This was the case in our patient, who developed perforation. The solution in these cases is positioning the lead on the interventricular septum, avoiding the RV anfractuous free wall.

\section{Conclusions}

In conclusion, this case is a very particular one. First of all, we've documented two distinct phases in the evolution of the ARVC (electrical phase - 2011 and structural phase - 2019). Moreover, by resuming endurance sports activity and in concordance with the literature data, we can only assume that the progression of the disease was accelerated and the arrhythmic risk was greater. Complications related to probe stability are common in this population, but myocardial perforation is a very rare and dangerous one. The therapeutic management of patients with ARVC has evolved over the years and continues to be an important challenge. To further improve risk stratification and treatment of patients, more information is needed on the natural history, long-term prognosis, and risk assessment.

\section{Consent}

Written informed consent was obtained from the patient for publication of this case report.

\section{Competing interests}

The authors declare that they have no competing interests. 


\section{References}

1. Marcus FI, McKenna WJ, Sherrill D, et al. Diagnosis of arrhythmogenic right ventricular cardiomyopathy/dysplasia: proposed modification of the task force criteria. Circulation 2010; 121(13):1533-1541.

2. Towbin JA, McKenna WJ, Abrams DJ, et al. 2019 HRS expert consensus statement on evaluation, risk stratification, and management of arrhythmogenic cardiomyopathy. Heart Rhythm 2019; 16(11):e301-e372.

3. James CA, Bhonsale A, Tichnell $C$, et al. Exercise increases age-related penetrance and arrhythmic risk in arrhythmogenic right ventricular dysplasia/cardiomyopathyassociated desmosomal mutation carriers. J Am Coll Cardiol 2013; 62(14):1290-1297.
4. Corrado D, Wichter T, Link MS, et al. Treatment of arrhythmogenic right ventricular cardiomyopathy/dysplasia: an international task force consensus statement. Eur Heart J 2015; 36(46):3227-3237.

5. Corrado D, Wichter T, Link MS, et al. Treatment of arrhythmogenic right ventricular cardiomyopathy/dysplasia: an international task force consensus statement. Circulation 2015; 132(5):441-453.

6. Wichter $\mathrm{T}$, Paul M, Wollmann $\mathrm{C}$, et al. Implantable cardioverter/defibrillator therapy in arrhythmogenic right ventricular cardiomyopathy: single-center experience of long-term follow-up and complications in 60 patients. Circulation 2004; 109(12):1503-1508. 